\title{
Un estudio de caso para analizar cómo ayudan los profesores en resolución de problemas matemáticos
}

\author{
A case-study for analyzing how the teachers help in \\ mathematical problem solving
}

\author{
Joaquín Fernández-Gago ${ }^{1}$ \\ José Carrillo Yáñez \\ Silvia María Conde Fernández ${ }^{3}$
}

\begin{abstract}
Resumen: Hay investigaciones que sostienen que influyen otras dimensiones además de las creencias respecto a la Matemática, Enseñanza de las Matemáticas y Aprendizaje de las Matemáticas en la actuación de profesores. Creemos que algunas de estas dimensiones pueden ser la idea de ayuda, de dificultad y comunicación que se usa con los alumnos. Analizamos con detalle una clase de la profesora Silvia para inferir cómo actúa en función de estas dimensiones. Para el análisis usamos la transcripción de una de sus clases con detalle. A través de este estudio perfilamos unos estilos en cuanto a las dimensiones estudiadas de algunos profesores españoles y, establecemos hipótesis de posibles inconsistencias de la profesora.
\end{abstract}

Palabras clave: ayuda, comunicación, resolución de problemas, estudio de caso.

Fecha de recepción: 4 de abril de 2017. Fecha de aceptación: 2 de octubre de 2018.

${ }^{1}$ Universidad de Málaga, jfernandezg@uma.es, orcid.org/0000-0002-0602-1334.

2 Universidad de Huelva, carrillo@ddcc.uhu.es, orcid.org/0000-0001-7906-416X.

3 Profesora de Enseñanza Secundaria del IES Guadalmedina, Málaga, España, ecomontilla@yahoo.es 


\begin{abstract}
Some investigations point out that, besides beliefs on mathematics and on mathematics teaching and learning, other dimensions' influence teachers' performance. Amongst these dimensions, we place the ideas of help, difficulty and communication, which is used with the students. One class by Silvia (a secondary teacher) is analyzed in detail in order to know how she performs against these dimensions. For this analysis we use the full transcription of the class. This study allows us to outline some styles in teaching of Spanish teachers depending on the studied dimensions. We also established hypothesis of possible inconsistencies in the performance of Silvia.
\end{abstract}

Keywords: help, communication, problem solving, case study.

\title{
1. INTRODUCCIÓN
}

Existen investigaciones en las que se pone de manifiesto que los profesores se proponen como metas tareas que fomenten la autonomía o trabajen la resolución de problemas matemáticos, que después no se llevan adelante, como por ejemplo Thompson (1984), Cohen (1990) o Xenofontos (2009), y otras como O'Shea y Leavy (2013). Más explícitamente, Schoenfeld (2015: 397) afirma:

La gente miente. Se mienten a sí mismos, y a los demás. Nunca puedes tomar lo que la gente dice (en entrevistas, respondiendo a cuestionarios, etc.) como valor seguro.

O'Shea y Leavy (2013) exponen, entre otros, el caso de Mike, uno de los profesores participantes en su estudio que manifestó una predisposición a usar resolución de problemas en el aula pero que en realidad no lo hizo, como demostraron los hechos siguientes: intervenía mucho guiando en exceso a los alumnos, no promovía la discusión entre los alumnos, daba pocas oportunidades de que los alumnos revisaran, examinaran sus dificultades y que desarrollaran estrategias de resolución de problemas. O'Shea y Leavy (2013:311) exponen:

También Mike menciona los compañeros de viaje en educación, incluyendo el equipo de dirección, los padres y los alumnos. Mike siente que debe haber resultados visibles para estos compañeros para atestiguar estos resultados y siente que con las prácticas constructivistas no obtendrán estos resultados. 
Xenofontos (2009:372), basándose en Raymod (1997), busca las explicaciones en el más allá de la Matemática, Enseñanza de las Matemáticas y Aprendizaje de las Matemáticas. Una dimensión que consideran es lo que tienes que hacer dentro de una cultura y un sistema educativo en el que los profesores son educados y trabajan, considerado esto como un todo. Este será nuestro punto de partida, considerar la idea de ayuda, la de dificultad y la comunicación con el alumno o comunicación que sugiere tenga el alumno consigo mismo como dimensiones relacionadas con lo que tienes que hacer dentro de una cultura y un sistema educativo, entre otras razones por las expectativas de los padres o por lo que manifiestan los compañeros sobre "qué hay que hacer". Creemos que, para enfrentarnos a estas dimensiones, los enfoques teóricos de Didáctica de la Matemática necesitan ser ampliados con otros como el de la inteligencia ejecutiva de Marina (2012), que puede verse resumido en Fernández-Gago y Carrillo (2014), donde se muestra la integración de los enfoques mencionados. La principal razón por la que usamos este marco es porque partimos de una definición de inteligencia que incluye la elección de las metas. En el marco teórico intentamos profundizar un poco más el por qué de esta elección.

O'Shea y Leavy (2013), con cada uno de los participantes, analizan si guían mucho o poco a los alumnos al resolver problemas, y estas guías determinan cómo se esfuerzan los alumnos. En Fernández-Gago y Carrillo (2014) y otro artículo en elaboración, reflejamos sendos estilos de los alumnos en cuanto a la forma de esforzarse en resolución de problemas: uno que llamaremos estilo Sísifo y otro que llamaremos estilo Ulises. El estilo Sísifo se caracteriza porque su inseguridad, por no disponer de un algoritmo que le dé la solución, le impide pararse a entender los problemas, rescatar recursos conocidos que le llevarían a la solución y comprobar las pocas conjeturas que formula, es decir, se trata de "un hacer por hacer", sin poner en juego las habilidades necesarias para que su esfuerzo sea eficaz. El estilo Ulises, por su parte, se caracteriza porque su gestión de la inseguridad, aun no teniendo el algoritmo que le dé la solución, le permite pararse a entender el problema, lanzarse a formular conjeturas y rescatar de su memoria recursos que le encaminan hacia la solución, es decir, se trata de un esfuerzo que pone en juego habilidades para ser eficaz. A este último tipo de esfuerzo le llamaremos esfuerzo creador. ¿Qué tipo de ayuda promueven los profesores para que se esfuercen los alumnos, hacia el estilo Sísifo o hacia el estilo Ulises? ¿Les propondrán problemas, y por tanto les invitarán a gestionar dificultades como hace Ulises? En la comunicación entre profesor y alumno, ¿ipromoverá ésta el esfuerzo creador? 
En este artículo abordamos cómo enfoca la profesora Silvia las dimensiones de ayuda, dificultad y la comunicación con el alumno y, lo hacemos observando y analizando con detalle una clase de la misma.

Los objetivos del artículo son:

- analizar cómo enfoca las dificultades de los alumnos para hacer por sí mismos un problema,

- indagar qué tipo de comunicación propone con sus alumnos, o propone para el alumno consigo mismo (habla interior), por ejemplo, si le incita a entender el enunciado, expresar conjeturas o si le incita a plantearse comprobaciones,

- analizar cómo gestiona la ayuda a los alumnos, observando el avance ante el problema, sobre todo cuando hay atascos o dudas,

- inferir predicciones sobre cómo propondrá a sus alumnos que se esfuercen ante un problema.

El artículo tiene los siguientes apartados: marco teórico, diseño de investigación y el modelo para el análisis de una clase, la profesora Silvia y su contexto, análisis y resultados y conclusiones.

\section{MARCO TEÓRICO}

En esta sección comenzamos resumiendo el marco teórico en el que nos basamos, esto es, la inteligencia ejecutiva de Marina (2004a) y Marina (2012), los estudios de Schoenfeld (1992), y caracterizamos las dimensiones que estudiamos: dificultad, ayuda y comunicación con los alumnos.

Existen definiciones de inteligencia que excluyen la elección de las metas y otras que separan inteligencia cognitiva y la emocional. Marina (2012:19), basándose en Marina (2004a), define la inteligencia de este modo: La capacidad de dirigir bien el comportamiento, eligiendo metas, aprovechando la información y regulando las emociones. Esta definición integra además de las emociones, aspectos cognitivos y la elección de metas. Establece, asimismo, dos niveles en la inteligencia, que son la inteligencia computacional y la inteligencia ejecutiva. Señala que la inteligencia computacional es la encargada de recibir información, elaborarla y producir respuestas con mayor o menor eficacia, y la inteligencia ejecutiva sería la inteligencia en acción, es decir, lo que un sujeto hace con sus 
capacidades. La misión de la inteligencia ejecutiva sería iniciar, dirigir y controlar las producciones de la inteligencia computacional. Más tarde Marina (2012:33) especifica las habilidades, que llama habilidades ejecutivas de la inteligencia ejecutiva: inhibir la respuesta, dirigir la atención, control emocional, planificación y organización de las metas, inicio y mantenimiento de la acción, flexibilidad, gestión de la memoria de trabajo y gestión de la metacognición. Desde estas definiciones, cualquier situación planteada con finalidad educativa, que propone una cuestión matemática, se acerca más a un problema cuanto más requiera el uso de las habilidades ejecutivas.

Marina (2009), valora el papel de las creencias en el aprendizaje porque, por ejemplo, afirma que debajo de sentimientos están actuando creencias implícitas, o porque a veces bloquean una de las funciones de la inteligencia que es conocer la realidad. Marina (2013, p.14) considera que en el quehacer de la enseñanza están los algoritmos y la heurística para aprender a ser creativos.

Schoenfeld (1992) establece cinco factores o variables que intervienen en el proceso de resolución de problemas: conocimiento específico, heurísticos, control, creencias y afectos, y prácticas, influyéndose unos con otros.

Existen paralelismos en cuanto al conocimiento, aprendizaje y el quehacer en la enseñanza de Schoenfeld y Marina. Ambos consideran que el conocimiento se compone de rutinas o algoritmos y de heurísticos, en la gestión de éstos influye el control o factor metacognitivo que gestiona que conocimientos o estrategias que intervienen en una tarea y por último ambos aceptan que hay creencias y emociones que intervienen en el proceso.

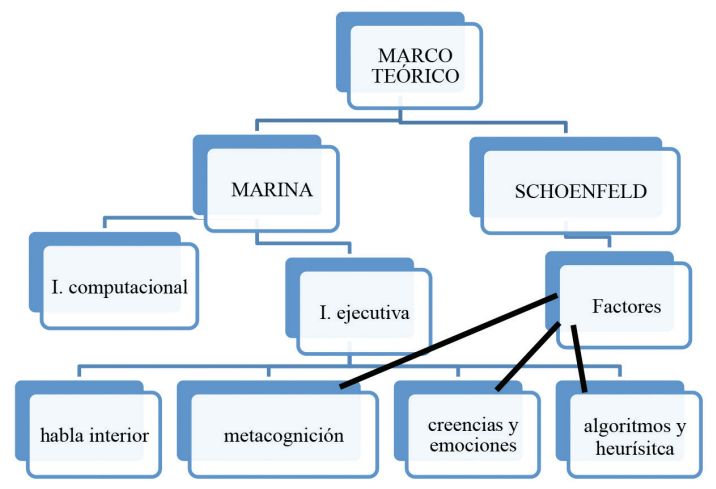

Figura 1. Perspectivas teóricas de Marina y Schoenfeld 
Exponemos a continuación como se concibe, desde la Didáctica de las Matemáticas y Marina las dimensiones estudiadas, para fijar cómo la concebimos.

La dificultad ha sido tratada como inherente al aprendizaje matemático y como conocimiento necesario de los profesores. Como inherente al aprendizaje matemático ha sido tratada por autores como Bouvier (1987), quien señala que en el aprendizaje se debe ir de lo complejo a lo simple pues es la complejidad la que le confiere significado. La complejidad en este sentido no es sinónimo de dificultad, pero implica dificultad. La consideración de la dificultad en Marina se resume en Marina (2004a):

El aprendizaje es recompensador cuando los padres del niño no se empeñan en suprimir las experiencias negativas, sino que le ayudan a superar su malestar, compartiendo su experiencia, y colaboran con él para que afronte las causas de la aflicción (p. 245).

Nosotros asociamos la idea de dificultad a la falta de conciencia de una destreza o recurso para que la situación a resolver pueda ser tratada con un procedimiento directo o algoritmo tal y como lo considera Marina.

Lo dicho en los párrafos anteriores influye en la idea de ayudar. Otros autores como Jaworski y Potari (2002) han tratado la idea de ayuda en la triada de enseñanza: gestión del aprendizaje, sensibilidad a los estudiantes y retos matemáticos. La idea de sensibilidad a los estudiantes tiene que ver con la idea de ayuda, que, tanto para Jaworski como para Potari, se basa en la expresada por Bruner (1985). Este autor presentó la idea de ayuda como metáfora del papel que juega en el aprendizaje el habla entre aprendices y expertos. Básicamente el experto debe ir guiando para ir fomentando su autonomía.

Nosotros consideramos que se puede ayudar a resolver problemas de distintas formas y para ello nos basaremos en el modelo de Schoenfeld. Recordemos que Schoenfeld (1992) establece 5 factores o variables que intervienen en el proceso de resolución de problemas: conocimiento específico, heurísticos, control, creencias y afectos, y prácticas, influyéndose unos con otros. Si un profesor ayuda a sus alumnos a que tenga más conocimiento específico o a que este esté mejor estructurado, ayudará al alumno a resolver problemas, como también si en su modelo de resolución de problemas es más capaz de usar heurísticos, y más aún si consigue que sus creencias sean más flexibles, y no una cortapisa, que les permitan o inciten a usar el conocimiento específico o heurísticos para resolver problemas. Así, podemos ayudar a un alumno suprimiendo una dificultad 
(para que no haya problema), o bien dirigiendo paso a paso un procedimiento que termina en la solución (sin necesidad de usar heurísticos y sólo usando ciertos conocimientos específicos), o bien forjando un modelo en el alumno para que aprenda a superar las dificultades (usando conocimiento específico y heurísticos, gestionándolos con el control), por ejemplo, cuando un alumno se encuentra atascado ante un problema

Ya se comentó en la introducción que analizamos qué tipo de comunicación establece la profesora con sus alumnos, y si ésta intenta que el alumno hable consigo mismo (habla interior) a través del lenguaje. El lenguaje se convierte en apoyo para nuestra autonomía, como establece Marina (2004b: 77): "El habla interior es el material del que están hechas nuestras voluntades". El lenguaje con uno mismo será potenciador, hacia la solución del problema, si es capaz de movilizar estructuras conceptuales, heurísticos o actitudes que el resolutor haya practicado antes en otros procesos de resolución de problemas. Gil, Blanco y Guerrero (2005) proponen un modelo de 4 fases para resolver problemas inspirado en el de Polya (1992), cada una de las cuales lleva asociadas autoinstrucciones, frases para decirse uno a sí mismo. Schoenfeld (1992) usa un lenguaje que consideramos potenciador en el apartado de la gestión y el control como factor metacognitivo, también con autoinstrucciones que expone el autor: ¿Qué estás haciendo? (¿Puedes tú describirlo con precisión?), ¿Para qué lo estoy haciendo? (¿Cómo encaja en la solución?), ¿Cómo me ayuda? (¿Qué harás tú con los resultados que obtengas?) (p.43).

Un lenguaje poco potenciador será el que nos desanime, nos dice que no podemos ir más allá, nos lleva a la pasividad, y a no ser capaz de comprender cierto malestar ante un problema. Para nosotros el lenguaje potenciador será aquel en el que, además de las auto-instrucciones de Schoenfeld (1992) mencionadas antes, el alumno entrena estas frases consigo mismo en un modelo de resolución de problemas: ¿Qué puedo hacer para entenderlo mejor?, ¿me lanzaré con esta conjetura o plan, aunque no esté seguro?, ¿es normal que esté agobiado o sufriendo, pero puedo hacer algo, por lo menos puedo pedir ayuda? ¿He visto una situación parecida? Si el profesor se comunica con los alumnos, intentando que el alumno tenga un habla interior, es lo que analizaremos en nuestra profesora. Si Marina le da importancia al lenguaje para nuestras acciones, las frases anteriores de Schoenfeld (1992) y las mencionadas en este párrafo son las concreciones del uso del lenguaje en resolución de problemas matemáticos 
Resumimos en la tabla 1 las distintas formas de concebir el habla interior en los autores.

Tabla 1. Formas de concebir el habla interior

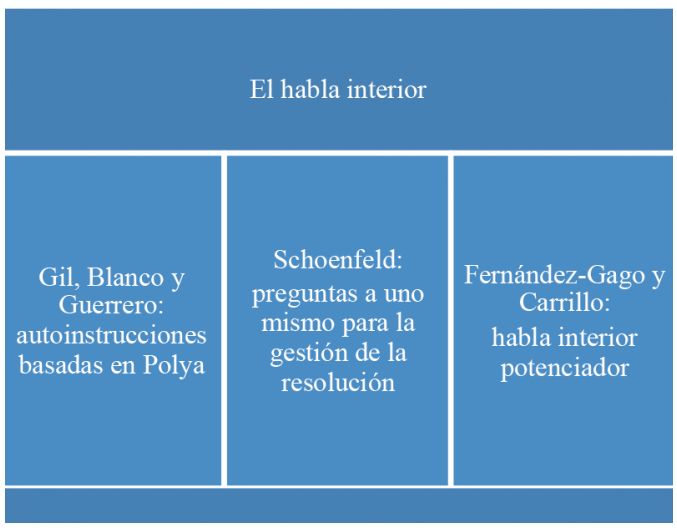

Resumiendo, la idea nuestra de dificultad está basada en la idea de dificultad de Marina, la idea de ayudar en los cinco factores que intervienen en la Resolución de Problemas de Schoenfeld, y la comunicación con los alumnos en las ideas de Marina y Schoenfeld.

\section{DISEÑO DE INVESTIGACIÓN Y MODELO PARA ANALIZAR LA CLASE}

\subsection{DISEÑO DE INVESTIGACIÓN}

Las dimensiones estudiadas son subjetivas para cada profesor y constituyen partes de una realidad que intentamos comprender de forma global. Intentamos describir y comprender formas de concebir el mundo educativo de un profesor particular, sin intentar buscar explicaciones generalizables, ya que no partimos de una realidad objetiva en el mundo educativo, sino de profesores y profesoras con una filosofía de la educación, unos conocimientos, unas metas y actuaciones que son difícilmente observables mediante la experimentación. Podríamos haber usado sólo cuestionarios que valoraran numéricamente las dimensiones estudiadas, pero la idea tan personal de la profesora, como veremos, respecto de los 
alumnos puede estar sustentada por creencias muy personales. Hemos usado la metodología cualitativa, ya que intentamos adentrarnos en la forma de entender la relación profesor-alumno en cuanto al uso de ayudas y comunicación. La profesora fue elegida de forma intencionada convirtiéndose en participante, y los resultados se han ido creando, en lugar de ser descubiertos, de forma coherente con un paradigma de investigación interpretativo. La razón para elegir el estudio de casos, procede del hecho de que las cuestiones cómo ayudar a los alumnos, cómo y qué exigirles, con qué intención comunicarles y cómo superar dificultades están sustentadas tanto por la "ética personal", como por la visión de inteligencia de la profesora, y este binomio ética-inteligencia es personal y "único", como su propia personalidad. Como teníamos interés, no sólo en analizar a la profesora, sino en posibles abstracciones, se trata de un estudio de casos particularista e instrumental, en el sentido de Muñoz-Catalán (2008), o inductivo en términos de Merriam (1998). Nuestro estudio de casos es descriptivo, ya que hacemos una rica y densa descripción de las interacciones que la profesora tiene con sus alumnos, interacciones que generalmente son alumno-profesor-alumno, en lugar de profesor-grupo.

Las fases de la investigación siguieron el siguiente proceso. En reuniones de profesores o cursos de formación del profesorado, pudimos constatar que una cuestión clave para ellos era el esfuerzo de los alumnos y cómo los profesores ayudan a éstos. En una revisión documental no encontramos datos al respecto en cuanto a educación matemática, por lo que decidimos entrar en el escenario y tomar datos mediante la realización de un cuestionario a 10 profesores de Secundaria en activo. El cuestionario constaba de preguntas de escala de valoración (1, 2, 3, 4; 1 poco de acuerdo y 4 muy de acuerdo) y preguntas abiertas, que se clasificaron en tres categorías: sentido de las actividades, sobre actitudes y valores y sobre la evaluación. Para el análisis de este cuestionario nos basamos en una tabla que calificaba las respuestas en aquellas que se sustentan en el fomento de la inteligencia ejecutiva o no la fomentan, y otra intermedia entre ambas, de tal forma que podían constituir un primer instrumento de investigación sobre la idea de ayudar y la dificultad. La profesora Silvia nos pareció una participante idónea, por ser buena informante y porque tanto en el cuestionario como en una posterior entrevista semiestructurada, que nos sirvió para confirmar respuestas o aclarar dudas, se declaraba firmemente convencida de promover la creatividad y autonomía de forma que el alumno supere dificultades inherentes al trabajo creativo más cercano al estilo Ulises. Así cabía la posibilidad de que Silvia representara el papel análogo al de Mike en O'Shea y Leavy (2013). 
Hecha ya la selección intencionada de Silvia, recogimos información en audio sobre la actuación de ella en 4 clases, de la que analizamos aquí una. Para el análisis y resultados, usamos estos instrumentos:

- entrevista previa de lo que Schoenfeld (2000) Ilama "la imagen de la lección", que constaba de qué metas se proponía, qué secuencia de acciones llevaría adelante para conseguir estas metas y qué dificultades preveía,

- pequeña entrevista al final para saber si había sido como ella tenía previsto, y consultar dudas,

- descripción detallada de lo que ocurrió en realidad,

- uso del modelo de Schoenfeld (2000) con adaptaciones de los autores para establecer episodios y subepisodios, así como metas y lenguaje usado,

- triangulación con expertos del grupo de investigación,

- entrevista final para aclarar dudas,

- elaboración del informe final.

\subsection{EL ANÁLISIS DE LA CLASE}

El análisis de la clase lo hicimos usando el modelo de Schoenfeld (2000), con adaptaciones de los autores. En él delimitamos un gran episodio, la clase entera, que se divide en subepisodios, que a su vez se vuelven a dividir en subepisodios, y así hasta una frase. Los episodios son conjuntos de acciones encadenadas que realiza la profesora, y, según Schoenfeld, Minstrell y Van Zee (2000), definidos por dos criterios: que tengan coherencia fenomenológica, y que tengan activada una meta de alto nivel (que vaya más allá de contenidos matemáticos). Algo singular de Silvia era que circulaba por el salón ayudando a los alumnos a resolver las tareas que les había planteado en días anteriores, y en cada grupo ayudaba en una tarea que no necesariamente era la misma que en otro.

Al haber una continua interacción de la profesora con alumnos individualmente al pasar por sus mesas, y que en estas interacciones la profesora podía volver al mismo alumno con el mismo problema, nos llevó a una primera adaptación de Schoenfeld (2000). Decidimos así que hubiera un gran episodio que fuera la propia circulación por la clase, dividido en subepisodios, y que cada uno de ellos fueran los problemas planteados que la profesora había mandado para clase o para casa, y que notamos por ( $\mathrm{p}-\mathrm{n}^{\circ}$ - de problema-página del libro). Cada uno se subdivide en subepisodios que corresponden a la interacción de un 
problema con un alumno (S1, S2, S3...), junto con el intervalo de tiempo en el que sucede. Al hacerlo de esta forma mantenemos los criterios de coherencia fenomenológica (en este caso los contenidos, el mismo problema), y que tiene que estar activada una meta de alto nivel (que el alumno sea activo).

La segunda variación que introducimos se refiere al tipo de comunicación. Antes de explicar cómo hemos etiquetado los tipos de comunicación, exponemos los esquemas de comunicación que observamos en la profesora, lo que nos encontramos en la realidad:

Primer esquema:

- el alumno pide ayuda,

- la profesora le dice lo que tiene que hacer, con exposición o explicación, y así va guiando al alumno a resolver la cuestión. Solicita del alumno una palabra o frase acertada. Cuando el alumno va escribiendo o diciendo algo la profesora confirma o no si va bien.

- la profesora se despide del alumno cuando ve que avanza hacia el objetivo de resolver el problema con el método que ella quiere, o éste manifiesta que lo entiende, que no tiene dudas.

Segundo esquema:

- el alumno pide que confirme o no, si va bien,

- la profesora le confirma o le critica la solución,

- por último, la profesora le sugiere un procedimiento directo para alcanzar la solución.

En ninguno de los dos casos la profesora induce al alumno a que hable consigo mismo, que hemos Ilamado habla interior, haciéndose cuestiones que le inciten a resolver por si mismo la cuestión, planteándose si puede usar heurísticos o no, si puede entrar uno u otro conocimiento o si puede gestionar de otra forma las actitudes que presenta ante la dificultad. Es, por tanto, este habla interior, una incitación al uso de la inteligencia ejecutiva.

Podemos encajar estos esquemas en la clasificación de Ribeiro, Monteiro y Carrillo (2009), como comunicaciones unidireccionales encadenadas, y en la de Schoenfeld, Minstrell y Van Zee (2000) la mayoría como IRE encadenados y existiendo también algunas del tipo elicitación socrática. Los IRE son comunicaciones en las que el profesor pregunta, el alumno responde y por último el 
profesor evalúa la respuesta del alumno y pasa a la siguiente cuestión. En la elicitación socrática hay bastante interacción entre profesor y estudiantes, en la que tanto la sustancia de la pregunta como la dirección provienen del profesor, de modo que éste es capaz de hilvanar las respuestas de los alumnos con las preguntas siguientes del profesor. Ahora bien, al ser la posibilidad de uso del habla interior (o sea, el lenguaje con uno mismo para usar la inteligencia ejecutiva) uno de los puntos importantes que estudiamos, no nos parece apropiada ninguna de las clasificaciones. En ambas se hace hincapié en el tipo de comunicación entre la profesora y el alumno, y sus efectos, mientras que nosotros necesitamos saber qué intención tiene la profesora de provocar la activación de las habilidades ejecutivas. Así necesitamos una clasificación que contemple la posibilidad de activar en el alumno un lenguaje que active su memoria para rescatar conceptos, procedimientos o actitudes que le ayuden a resolver el problema. Por tanto, nos hemos decidido por la clasificación: comunicación que provoca inteligencia computacional y que no necesita usar el habla interior, la que provoca inteligencia computacional con necesidad del habla interior, y, por último, la que necesita inteligencia ejecutiva, que necesariamente implica uso de habla interior. A algunas interacciones entre la profesora y los alumnos del tipo comunicación que provoca inteligencia computacional y no necesita habla interior, le hemos añadido la expresión habla simple, ya que creemos que es bastante sugerente para el lector.

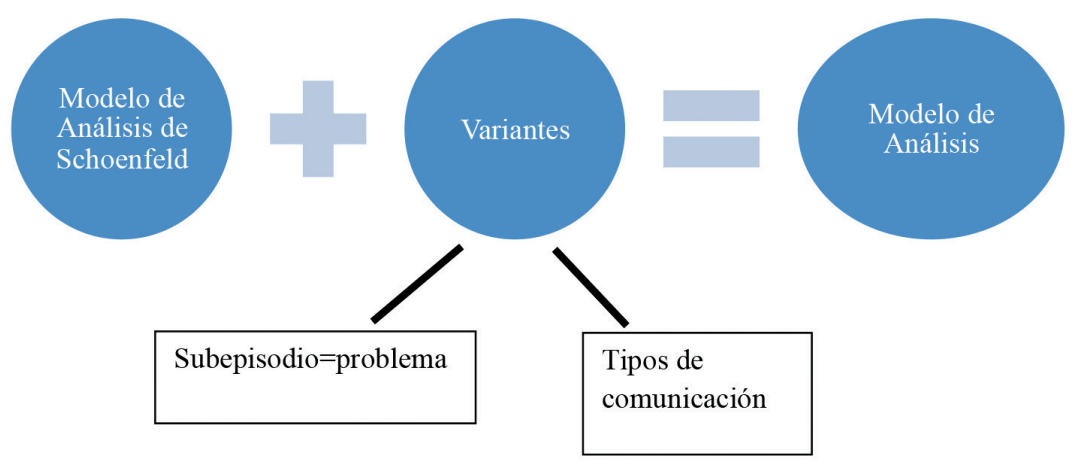

Figura 2. Modelo de Análisis 


\subsection{ALGUNAS DE LAS VISIONES DE LA PROFESORA SOBRE LA ENSEÑANZA Y APRENDIZAJE DE LAS MATEMÁTICAS}

La profesora impartía Matemáticas a alumnos de 11 a 13 años. En el Departamento de Matemáticas del centro donde trabaja la profesora, se intenta instruir en resolución de problemas desde principio de curso, dedicando la primera unidad didáctica a conocer actitudes y heurísticos de 3 fases de resolución de problemas que figuran como "tema 0" del libro: Comprensión del problema; Lleva adelante tu estrategia y Comprueba tu solución. Aunque el libro recomienda seguir trabajando estos heurísticos en los siguientes temas, sólo tenemos constancia de que la profesora lo hiciera en el "tema 0". Hay que mencionar que el currículo vigente cuando la profesora impartió esas clases incluía la resolución de problemas como algo importante, incluso como contenido. Concretamente en la Orden de 10 de agosto de 2007 en la página 51 incluye como primer Núcleo temático la resolución de problemas como núcleo transversal, y en la página 52 expone:

\section{Contenidos relevantes}

El alumnado de esta etapa educativa debe conocer y utilizar correctamente estrategias heurísticas de resolución de problemas, basadas, al menos, en cuatro pasos: comprender el enunciado, trazar un plan o estrategia, ejecutar el plan y comprobar la solución en el contexto del problema.

Interacción con otros núcleos temáticos y de actividades

Más que estar relacionado con el resto de núcleos temáticos de matemáticas, la resolución de problemas constituye en sí misma la esencia del aprendizaje que ha de estar presente en todos los núcleos temáticos.

Con respecto al cuestionario y entrevista posterior a él, vemos que sobre sus afirmaciones acerca de la dificultad, ayuda y habla interior se manifiesta muy favorable a promover la inteligencia ejecutiva, sobre todo por su defensa de que el alumno sea creativo y ésta se puede aprender (“... la creatividad es algo que se tiene, yo pienso, y, pero si tú la vas fomentando, pues esa creatividad va creciendo, pero si no, yo creo que llega un momento en que a lo mejor la vas dejando de lado"), por considerar importante la resolución de problemas, por 
señalar que al alumno se le ayuda de forma que consiga pensar los problemas por sí solo ("con las indicaciones que yo le he dado, que ellos piensen por sí solos"), que debe descubrir ("Ia preguntas sobre a qué se refiere cuando habla de madurez y crecimiento]... pues descubrir otra manera de hacer las cosas, antes no se planteaban descubrir la solución"), que aprenda por sí mismo, que hay diferentes formas de afrontar una misma situación ("sirve para su vida [se refiere a un alumno genérico] con vistas a que cuando tengan dificultades, a lo mejor en su vida intenta buscar otros caminos, otras formas de hacer las cosas"), que se-sea comprensivo con sus dudas([ante un alumno que sigue manifestando dudas] “...párate, relájate, no pasa nada, con lo que ya te he dicho piensa en eso, deja que pase el tiempo ahora, ...llega a tu casa y esta tarde te sientas, lo piensas y lo miras de nuevo con otros ojos a ver si lo ves de otra manera..."), o que debe aprender de los errores ("yo siempre aprendí de los errores, siempre, entonces, si hay un error, se puede transformar"). No nos queda claro si su visión tiende a promover la inteligencia ejecutiva cuando dice que los alumnos "no se deben sentir solos ante la dificultad de un problema", ante las dudas cuando manifiesta que se lo explica una y otra vez a los alumnos. Ante su visión sobre el habla interior (al preguntarle sobre si cree que los alumnos tienen o usan algún tipo de habla interior al resolver problemas, manifiesta que "ni idea, no lo sé"), sobre la forma de mejorar resolviendo problemas (al preguntarle si un modelo le puede ayudar, manifiesta que "se mejora con esfuerzo, con ganas", o al preguntarle sobre qué es ayudar, manifiesta que "es estar cerca del alumno en todo momento... que no se vea solo ante la dificultad de un problema..."). En la entrevista sobre el cuestionario ella terminó manifestando, a petición propia, lo siguiente:

Silvia: Lo más importante para mí es querer a los alumnos, desde el primer día, y quererlos con sus virtudes, con sus defectos, con su todo, intentar que ese cariño les ayude a aprender matemáticas, desde ahí, desde el cariño que yo les tengo yo les puedo ayudar, ellos se sienten confiados, se sienten tranquilos,

Resumiendo, podríamos decir, que la profesora parece, por lo visto con los datos recogidos, lo siguiente:

- intenta que su papel sea el de inducir y promover la acción de los alumnos, 
- considera como papel del alumno la investigación y la resolución del problema tal y como prescribe el currículo,

- en cuanto a su idea de ayudar está más bien cercana a sí promover ayuda, habla interior y gestión de las dificultades tendentes a fomentar el esfuerzo creador (propio del estilo Ulises), aunque con algunas dudas.

Para resolver las dudas se grabaron varias clases.

\section{ANÁLISIS Y RESULTADOS}

Como hemos dicho, analizamos aquí solo la segunda clase de las 4 grabadas. El análisis lo hemos dividido en: 4.1 La imagen de la lección y como fue en realidad, 4.2 Los episodios desgranados y 4.3 Resultados.

\subsection{LA IMAGEN DE LA LECCIÓN Y COMO FUE EN REALIDAD}

La profesora había dado una clase con este grupo de 1ำ de ESO a las 8:15 de la mañana, y ahora pretendía continuarla a las 10:15, que es la clase que analizamos. Si en la clase anterior los alumnos trabajaron problemas susceptibles de resolverse con ecuaciones, nada más comenzar Silvia Conde escribió en la pizarra los problemas para esta hora: 27, 28, 30, 31 y 324 de la página 198 del libro de texto que aquí reproducimos.

Problemas para resolver con ecuaciones

27. Si triplicas un número y al resultado le restas 16, obtienes 29. ¿Cuál es el número?

28. ¿Cuál es el número que sumado con su anterior y su siguiente da 117 ?

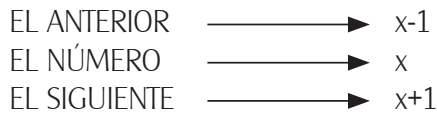

${ }^{4}$ La página de estos problemas está en el Anexo. 
30. Si a un número le restas 28 unidades, obtienes el mismo resultado que si divides entre 3. ¿Qué número es?

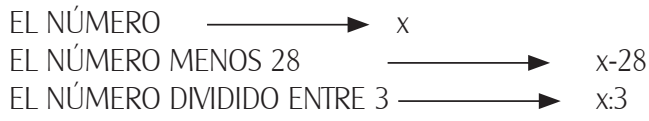

31. Si a este cántaro le añadieras 13 litros de agua, tendría el triple que si le sacara dos. ¿Cuántos litros hay en el cántaro?

32. En mi colegio, entre alumnos y alumnas somos 624 . El número de chicas supera en 36 al número de chicos. ¿Cuántos chicos hay? ¿Y chicas?

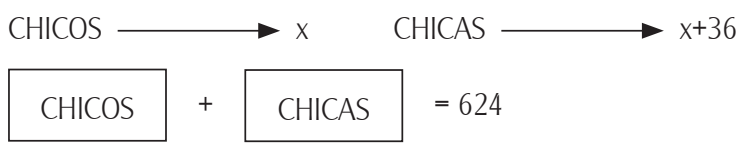

Así que la imagen de la lección, según nos comentó en una entrevista previa constaba principalmente de la siguiente secuencia de acciones:

- preguntar si hay dudas de los alumnos a la hora de resolver ecuaciones,

- usar el script o guion de explicar las dudas de los alumnos, o analogías del tipo "pera con plátanos no se pueden sumar", "sumamos las x con las x", el igual es una barrera", "Io que está multiplicando pasa dividiendo", y

- dar por terminado un problema cuando el alumno manifieste que no hay dudas, que lo entiende, poner positivos a los que le muestren el problema bien hecho.

En cuanto a las dificultades que ella imaginaba estaban las de resolver ecuaciones a la hora de reducir expresiones y transponer términos, y a la hora de plantear los problemas mediante ecuaciones. No nos especificó qué tipo de dificultades habría específicamente con cada alumno. También pensaba que habría alumnos que no se esforzarían lo suficiente, y que ella-tendría que incitarlos a trabajar. Nos comentó que ella consideraba importante la comprobación de las soluciones y fomentar la autonomía de los alumnos.

Tras terminar la clase le preguntamos si llevó adelante las secuencias de acciones que tenía previsto, y si las dificultades eran las esperadas. También le 
preguntamos si los positivos que puso al principio de la clase, sobre ecuaciones mandadas para casa, si los ponía con observar que estaban bien.

Lo que ocurrió en realidad, se entiende mejor con la distribución de los alumnos en el aula, que notamos por S1, S2...S24.

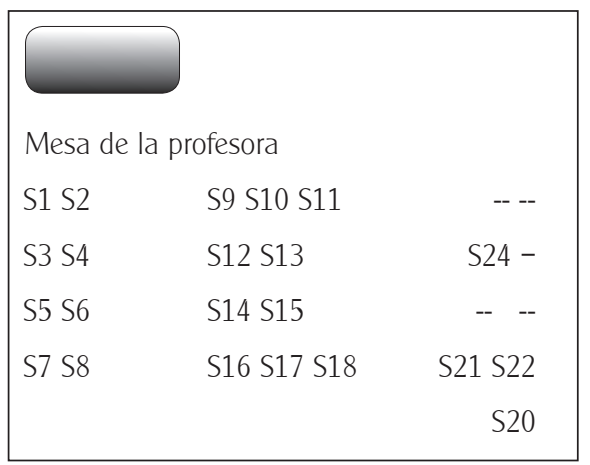

Figura 3. Distribución de los alumnos en la clase.

La profesora circuló por el aula interactuando con cada alumno, siendo esta interactuación igual ante un mismo problema, sin influir el alumno en cuestión. La ayuda a los alumnos que la piden hace que los alumnos trabajen, aunque nunca comprueban. La mayoría de esquemas de comunicación usados por Silvia Conde fueron del tipo comunicación que provoca inteligencia computacional y que no necesita usar el habla interior. Vemos aquí una diferencia entre la imagen de la lección y lo que ocurrió en realidad.

Para al análisis desgranado en episodios y subepisodios, exponemos las metas que observamos en clase:

M1: plantear ecuaciones para problemas que se resuelven con ellas,

M2: aplicar los procedimientos para resolver ecuaciones,

M3: autoconfianza para hacer las tareas por sí mismos,

M4: que sean críticos,

M5: que los alumnos se esfuercen,

M6: mantener la convivencia en el aula,

M7: organizar la clase.

A continuación, figura la división de la clase en episodios y subepisodios. 


\begin{tabular}{|c|c|c|c|c|c|}
\hline \multirow[t]{9}{*}{$\begin{array}{l}{[1]} \\
\text { La lección } \\
\text { completa }\end{array}$} & $\begin{array}{l}{[1.1]} \\
{[0-10: 40]} \\
\text { Introducción } \\
\text { y tareas } \\
\text { administra- } \\
\text { tivas }\end{array}$ & \begin{tabular}{|l} 
[1.1.1] [0-3:05] \\
Introducción callar, \\
sentar y \\
comentarios
\end{tabular} & & & \\
\hline & \multirow{6}{*}{$\begin{array}{l}\text { [1.2] [10:50- } \\
\text { 52:25] } \\
\text { Resolver } \\
\text { problemas } \\
\text { usando } \\
\text { ecuaciones }\end{array}$} & [1.2.2] p-6-195 & $\begin{array}{l}\text { [1.2.2.1] [10:50-13:08] } \\
\text { Corrige a S17 }\end{array}$ & \multicolumn{2}{|c|}{$\begin{array}{l}\text { [1.2.2.2] [43:55-46:00] } \\
\text { Corrige a S6 }\end{array}$} \\
\hline & & $\begin{array}{l}\text { [1.2.3] alumnos sin } \\
\text { trabajar o } \\
\text { molestando }\end{array}$ & $\begin{array}{l}\text { [1.2.3.1][10:20-10:50] } \\
\text { Incitación al } \\
\text { esfuerzo a S9 }\end{array}$ & $\ldots$ & $\begin{array}{l}\text { [1.2.3.8][41:45-42:30] } \\
\text { iA trabajar! a S21, } \\
\text { S22 y S23 }\end{array}$ \\
\hline & & [1.2.4] p-3-195 & $\begin{array}{l}\text { [1.2.4.1] [13:50-15:05] } \\
\text { Dirige a S13 }\end{array}$ & \multicolumn{2}{|c|}{$\begin{array}{l}\text { [1.2.4.2][30:10-31:15] } \\
\text { Dirige a S12 y S13 }\end{array}$} \\
\hline & & [1.2.10] p-29-198 & $\begin{array}{l}\text { [1.2.10.1] [33:50- } \\
\text { 34:44] Dirige a S14 }\end{array}$ & \multicolumn{2}{|c|}{$\begin{array}{l}\text { [1.2.10.2] [37:45-38:20] } \\
\text { Dirige e incita a } \\
\text { comprobar a S14 }\end{array}$} \\
\hline & & & $\begin{array}{l}\text { [1.2.11.1] [38:20- } \\
\text { 40:40] Dirige a S4 }\end{array}$ & $\ldots$ & $\begin{array}{l}\text { [1.2.11.4][43:55-44:20] } \\
\text { Corrige y dirige a S3 } \\
\text { y S4 }\end{array}$ \\
\hline & & [1.2.12] p-9-195 & $\begin{array}{l}\text { [1.2.12.1][50:38- } \\
\text { 51:25] Dirige a S16 y } \\
\text { S17 }\end{array}$ & & \\
\hline & \multirow{2}{*}{$\begin{array}{l}\text { [1.3] [47:20- } \\
55: 10] \\
\text { Cierre de la } \\
\text { clase }\end{array}$} & $\begin{array}{l}\text { [1.3.1] } \\
\text { Pone positivos }\end{array}$ & $\begin{array}{l}\text { [1.3.1.1] [47:20-47:25] } \\
\text { Pone positivos a S1 }\end{array}$ & \multicolumn{2}{|c|}{$\begin{array}{l}\text { [1.3.1.2] [51:25-51:37] } \\
\text { Pone positivos a S5 } \\
\end{array}$} \\
\hline & & $\begin{array}{l}\text { [1.3.2] Deja salir y } \\
\text { no deja salir }\end{array}$ & $\begin{array}{l}\text { [1.3.2.1] [50:10-50:38] } \\
\text { Deja salir sólo con } \\
\text { deberes terminados }\end{array}$ & \multicolumn{2}{|c|}{$\begin{array}{l}\text { [1.3.2.2] [51:37-55:10] } \\
\text { Deja salir sólo con } \\
\text { deberes terminados }\end{array}$} \\
\hline
\end{tabular}

Figura 4. La clase entera 


\subsection{RESULTADOS}

La triangulación la llevamos a cabo del siguiente modo: tras recabar los datos por el investigador 1, éste pasaba un informe al investigador 2, que tras $90 \%$ de consenso se daba por válido. A continuación, se exponían los resultados ante el grupo de investigación, con expertos, que servía para matizar resultados o desecharlos.

Una apreciación global que tenemos en cuanto a la profesora es su gran empatía con los alumnos, lo que hace que ellos muestren una gran confianza en acudir a ella para que les ayude, hecho que no hemos percibido con otros profesores a cuyas clases hemos asistido.

Otra apreciación global que nos sugiere la clase en cuanto a la metodología, es que es como nos esperábamos, como nos decía la encuesta y la entrevista que iba a suceder, intentando ayudar acompañándolos, animándolos, minimizando la importancia del error si no salía el problema. En cambio, nos extrañó al principio que los alumnos pudieran levantarse, hacer mucho ruido, y algunos no trabajar, usando como plan de acción para solventarlo sólo las buenas maneras, como nos comentó en una entrevista posterior. Esto, sin duda, nos extraña menos una vez que hemos entrado a fondo en el mundo personal de Silvia, y hemos comprendido lo que es para ella ayudar.

En cuanto a la finalidad de la asignatura, nos parece que da importancia a la resolución de problemas (y por tanto parece que busca fomentar las habilidades ejecutivas), ya que en las tareas de la clase ha propuesto varios problemas, susceptibles todos de resolverse con ecuaciones. Otra cuestión es que intente seguir instruyendo el modelo de resolución de problemas tal y como prescribe el currículo en cuanto al modelo de cuatro pasos y a su interacción con el núcleo temático de Álgebra. Un ejemplo donde esto se pone de manifiesto, es en el episodio [1.2.9] que se refiere a todas las interacciones con el problema (p-30198). Este es un problema que se puede resolver haciendo un gráfico (el tema 0 de resolución de problemas que ella trabajó al principio de curso contiene uno igual), o con fracciones (el tema 7 de fracciones tiene algunos muy parecidos, como el 30 o el 3o de la página 141 del libro de texto), o también con una particularización organizada y sistemática o buscando un problema familiar. En la entrevista final, concretamente en las preguntas 1, 2 y 3, nos aseguró que había hecho y corregido los problemas de las páginas anteriores. En los subepisodios [1.2.9.1], [1.2.9.2], [1.2.9.3] y [1.2.9.6] no contempla ninguna de estas alternativas, sino que el objetivo de resolver los problemas con ecuaciones está por 
encima del de promover las habilidades ejecutivas. Exponemos como ejemplos los subepisodios [1.2.9.1], [1.2.9.3], [1.2.9.4] en los que interacciona con el alumno S4.

La profesora usa aproximadamente 11 minutos en intentar callar a los alumnos, poner positivos a algunos alumnos como S4 (episodio [1.1]), anima a los alumnos a trabajar y les anuncia la tarea, que será resolver problemas que están en el libro de texto al final del tema de ecuaciones. La alumna S4, tras estos 11 minutos, trabaja algunos problemas como el problema (p-28-198), y le solicita ayuda a la profesora hacia los 18:30. Lo resuelve y se enfrasca con el problema (p-30-198), pidiéndole ayuda hacia el minuto 28:

[1.2.9.1] Corrige y dirige a S4

La alumna S4 la Ilama. Le muestra en su papel el planteamiento del problema (p-30-198) que viene en el libro:

Un número: $x$

Un número menos 28: $x-28$

Número dividido entre $3: \mathrm{x}-28=\mathrm{x}: 3$

y se establece un diálogo entre $\$ 4$ y Silvia:

1. S4: ¿Y esto ahora cómo sería? ¿Sería x por x? (señalando tanto la x que está en un miembro como la que está en el otro).

2. Silvia Conde: Pero es que en vez de dividir... (se para a pensar). Vamos a ver ¿Qué vas a hacer $\mathrm{S} 4$ las multiplicaciones y las divisiones o las sumas y las restas?

3. S4: Las multiplicaciones y las divisiones.

4. Silvia Conde: Si éste está aquí dividiendo (señalando con el dedo al 3 del miembro derecho) pasa...

5. S4: iAh vale!

6. Silvia Conde: multiplicando, y lo multiplica a todo.

7. S4: Vale

8. Silvia Conde: Este es un poco difícil.

En la línea 2 vemos como intenta recordarle el algoritmo para resolver ecuaciones con denominadores, y activa para ello las metas M2 y M5. Entre las líneas 4 a 6, al decirle claramente que pasa multiplicando, lo cual ella cree que va a tranquilizar a la alumna y hace que no se sienta sola. La comunicación de la profesora con la alumna es una comunicación que provoca inteligencia 
computacional, sin necesidad de habla interior. Creemos que ella consigue que los alumnos que piden ayuda se esfuercen por aprender el método de planteamiento y resolución de ecuaciones de primer grado activando las metas M2 y M5. También nos parece que se aleja de los que prescribe el currículo en cuanto a resolución de problemas ya que se le suprime la dificultad con la ayuda que le da, y que podría haberse apoyado en otro tipo de comunicación como: ¿qué hiciste en situaciones parecidas? o ¿es malo que estés atascada? Creemos que esto la hubiera acercado más a fomentar el esfuerzo creador del estilo Ulises.

La profesora sigue circulando por la clase y le reclama la ayuda S4, otra vez, hacia el minuto 32:30:

\section{[1.2.9.3] Dirige a S4}

La profesora viene de ayudar a S1 con el mismo problema (p-30-198), comentándole al final que éste era muy complicado, y que no tendría que haberlo puesto. En la misma posición que atiende a S1 atiende a S4.

1.S4: ¿Esto sigue así? (le pregunta la alumna tras mostrarle en su papel)

$$
28-3=x-x
$$

$89=(-1) x$

$\mathrm{x}=89{ }^{*}$ ) [aquí podría haberla dejado que se equivocara y comprobara]

2. Silvia Conde: Ves, pero espérate S4. ¿Cómo lo hacemos? (Le escribe en su papel lo siguiente

$$
\begin{aligned}
& 3(x-28)=x \\
& \text { (y le dice) }
\end{aligned}
$$

Vale. A mí me gusta más con las rayitas de fracciones. Este 3 (señalando al del miembro de la derecha) pasa multiplicando y lo multiplica a todo. Sería tres por $\mathrm{x}$ menos 28 igual a x. ¿Vale? Y ahora sigue tú trabajando la ecuación. (Dirigiéndose a nosotros nos dice: es que le pregunté a otra profesora del Departamento, y me dijo que los problemas con fracciones mejor para $2^{\circ}$ ).

En la línea 1, cuando escribe la solución x=89, la ayuda de la profesora va encaminada a que repita el procedimiento de forma correcta. Activa aquí las metas M2 y M5. Vemos que la profesora ha conseguido que la alumna 
mantenga interés por aprender a resolver el problema, a pesar de las dificultades o errores. Otra cuestión es cómo ha gestionado estas dificultades o errores pues la alumna no ha tenido que comprobar si 89 es el número buscado porque la ayuda es darle el siguiente paso del proceso correctamente. En cuanto a la comunicación es aquella que provoca inteligencia computacional sin que provoque habla interior, como por ejemplo, ¿Cumple 89 lo que queremos? 0 ¿Es malo equivocarse en Matemáticas? Por lo anterior, creemos que está lejos de promover el modelo de 4 pasos que prescribe el currículo y así se aleja de promover el esfuerzo creador del estilo Ulises.

La profesora sigue circulando por la clase ayudando a otros compañeros, con resolver este problema u otros, y at hacia el minuto 40, tras llamarla S4, se dirige a ella otra vez:

[1.2.9.4] Dirige a S4

La alumna le muestra la resolución de la ecuación, con un paso más desde donde se quedó en el subepisodio anterior:

$$
\begin{aligned}
& 3(x-28)=x \\
& 3 x-84=x
\end{aligned}
$$

1.S4: ¿Y ahora cómo va?

2. Silvia Conde: ilas " $x$ " con las " $x$ ", S4!

3. S4: Sería $4 x$, ¿no?

4. Silvia Conde: No

5. S4: ¿Cuatro?

6. Silvia Conde: No

7. S4: tres $x$ menos $x$

8. Silvia Conde: Tres $x$ menos $x$, muy bien. (Escribe la alumna $3 x-x$, y se para). Igual (la alumna escribe el igual a continuación de $3 x-x$, quedando $3 x-x=y$ le confirma que va bien). Muy bien. (Ia alumna continúa escribiendo el 84, quedando $3 x-x=84$ ). Muy bien. ¿Cuánto es tres $x$ menos x? 2x (La alumna escribe $2 x=84$ y Silvia Conde le confirma que va bien). Muy bien. ¿Cuánto vale la x?

9. S4: ochenta y 4 entre 2 (Escribe 84:2).

10. Silvia Conde: Una vez que llegas aquí (señalándole la ecuación $x-28=x: 3$ ), las " $x$ " con las " $x$ " y los números con los números. 
La alumna en la línea 1 quiere que la profesora le diga cómo seguir, a lo que la profesora le responde rescatándole de la memoria el procedimiento para resolver ecuaciones (activa las metas M2 y M5), pues cree que ayuda más a la alumna sin que esta llegue a sentirse insegura y por eso le suprime la dificultad. La profesora ha conseguido que esta alumna mantenga su interés por aprender a resolver el problema y llegar hasta el final. Como en los Análisis anteriores se aleja de la prescripción del currículo de que el alumno utilice estrategias heurísticas en cuatro pasos, con incitaciones a la comprobación o con preguntas. para cuando esté atascada, del tipo ¿Qué haces para resolver las del primer grado? Por lo anterior pensamos que está más cerca de promover el estilo Sísifo que el estilo Ulises.

La dificultad que aparece en este subepisodio, podría analizarse con Papini (2003), cuando afirma que:

La aparición de estas dificultades puede tener relación... pero también, con fenómenos de tipo didáctico como suele ser el excesivo énfasis puesto, en las clases de matemática, en la mecanización del trabajo algebraico en desmedro de un uso modelizador de estas herramientas (p.45).

El análisis que hemos hecho de los tres subepisodios se confirman en el resto de subepisodios [1.2.n] donde ella actúa siempre de la misma forma. Le pedimos a la profesora el examen de este tema, y por las preguntas que ponía, resolver ecuaciones y un problema susceptible de resolverse con ecuaciones y la forma de puntuarlo, nos confirmó se valoraba el algoritmo. Tras analizar estas dimensiones creemos que el tipo de esfuerzo que fomenta no tiende a ser un esfuerzo creador. En las dos clases siguientes que analizamos a Silvia Conde, que fueron de Geometría, nos confirmaron los resultados, tanto a los dos investigadores como a los expertos.

A los alumnos de esta clase se les planteó el mismo problema en la prueba inicial del curso siguiente, unos 4 meses más tarde, obteniendo unos esquemas de resolución muy superficiales, con algunos planteamientos que parecían decirnos que el alumno intuía que se podía resolver con ecuaciones, pero sin llegar a plantearla bien y no resolviéndola correctamente. 


\section{CONCLUSIONES}

En primer lugar, al haber comprobado que las dimensiones estudiadas están influyendo en las actuaciones de profesores, creemos que esto valida el marco teórico que presentamos para el análisis de profesores, destacando los conceptos inteligencia ejecutiva y esfuerzo creador. Como hemos comentado, uno de los problemas que presentan los estudios sobre Dominio Afectivo es que cada estudio presenta un marco teórico distinto, entre otras razones por la dificultad de caracterizar constructos como actitud o motivación, por ejemplo. Nuestro marco comienza por definir antes la inteligencia (que nos ha permitido caracterizar dimensiones), primer paso para afrontar otros constructos propios del dominio afectivo.

Una segunda conclusión es que las dimensiones dificultad, ayuda y comunicación con los alumnos determinan una forma de actuar de la profesora que parece influir en una forma de actuar de los alumnos. Que la alumna S4 no conviva con el error, no compruebe, su gestión de las dudas se reduzca a preguntar a la profesora, o no rescate de su memoria recursos que sí tiene disponibles nos parecen estar influidos por la actuación de la profesora. La profesora, a su vez, nos parece estar bastante influida por la idea de que ayudar es no dejarlos solos, acompañarlos en todo momento y quererlos, como ella afirma. Así parece inferirse que la profesora presenta una inconsistencia entre su idea de fomentar la creatividad, autonomía, hacer las cosas de otras formas o aprender de los errores, no así ante la gestión de la duda, en la que manifestaba que era importante que los alumnos no se sintieran solos, que es lo que ella ha hecho. Este caso puede ser un caso representativo de lo que ocurre en las aulas de España, donde los profesores manifiestan unas intenciones tendentes a que los alumnos sean creativos y críticos, pero que en realidad la forma de actuar sea muy tradicional, es decir, los profesores aspiran al estilo Ulises y quizás se queden en el estilo Sísifo.

El hecho de que su forma de ayudar sea básicamente la de dirigir usando un procedimiento que desemboque en la solución nos lleva a pensar que no se implementan heurísticos, como los considerados en Schoenfeld (1985) o en Puig (1992). También podríamos discutir acerca del uso de esta forma de ayudar centro del contexto del Álgebra. En Godino (2003) se consideran 6 etapas en el uso de símbolos algebraicos: letra evaluada, letra usada como objeto, letra ignorada, letra usada como incógnita específica, letra usada como número generalizado y letra como variable. Las ayudas sugeridas por la mayoría de los profesores 
en Secundaria alejan al alumno de considerar la letra como número generalizado, y más aún como variable. Otros autores, como Saiz y otros (2014), critican el énfasis en las destrezas para plantear y resolver ecuaciones en el aprendizaje del álgebra, además de poner de relieve la complejidad en la gestión de una clase de álgebra. Esto puede tener implicaciones en el modelo de formación del profesorado en España.

Aunque nuestro estudio no es sobre creencias de los profesores, por lo expresado por Silvia en cuestionarios y entrevistas, además de lo observado, nos hace intuir que las dimensiones de ayuda, dificultad y comunicación con los alumnos podrían ser "hitos" que nos dirijan hacia una búsqueda de sentido (Leatham, 2006) ante la aparente inconsistencia entre su idea de ayudar y las otras dimensiones, lo cual debería ser confirmado en una investigación posterior. Aquí reside una de las relevancias de nuestro estudio, pues creemos que puede explicar, al menos en parte, las inconsistencias de profesores en España, pues muchos profesores en España manifiestan en sus programaciones que intentarán que sus alumnos sean creativos y críticos y en realidad sus prácticas son tradicionales. Una posible explicación de lo que ocurre está en que quizás para la profesora lo que tiene que hacer está en ayudar como ella ayuda para superar dificultades.

En tercer lugar, el modelo de análisis adaptado de Schoenfeld (2000) es operativo para el análisis de profesores que circulan por la clase y que han propuesto más de una tarea a todos sus alumnos. Como hemos dicho, el modelo propuesto admite más posibilidades, en los siguientes sentidos:

- en el modelo los problemas son los que definen los subepisodios, hecho que puede cambiar definiendo los subepisosdios por alumno, que puede ser interesante para analizar qué perfil tiene ese alumno o que difícil es ese problema para esa clase,

- el modelo, definiendo el problema, el subepisodio se puede representar gráficamente en un gráfico cartesiano, en el que el eje horizontal representa a los alumnos y en el vertical el tiempo en el que empiezan a interactuar la profesora con el alumno. 


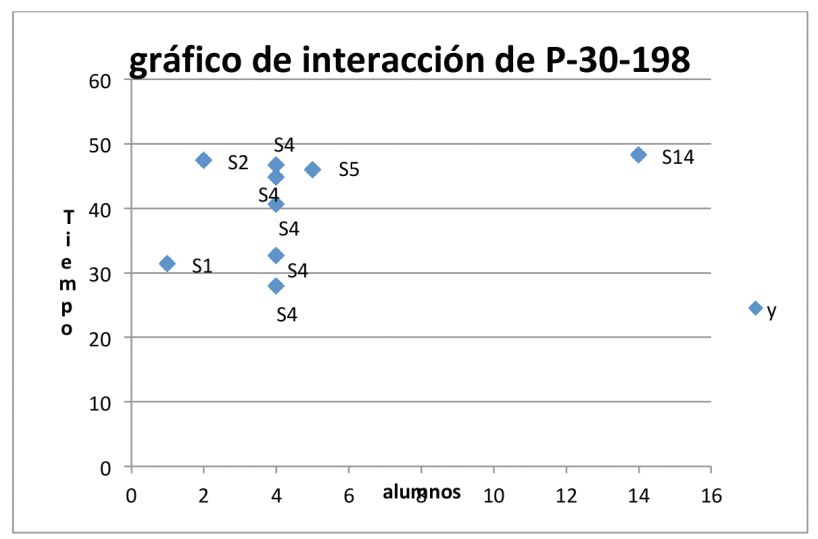

Figura 5. Gráfico de interacción de P-30-198. Al alumno que más acude a ayudar es a S4.

Además, para un alumno determinado, $S_{i}$, podemos confrontar las variables problemas y tiempo para saber si dedica mucho o poco tiempo a cada problema, o confrontando las variables problemas y alumnos, para ver qué alumnos se enfrascan en más problemas o menos.

\section{Agradecimientos}

Damos las gracias a la profesora Silvia Conde por permitirnos entrar en sus clases y tomar datos necesarios para esta investigación.

\section{REFERENCIAS}

Aguirre, J. \& Speer, N. M. (2000). Examining the relationship between beliefs and goals in teacher practice. Journal of Mathematical Behavior, 18(3), 325- 356. doi: 10.1016/ S0732-3123(99)00034-6.

Ball D.L. y Otros (2008). Content knowledge for Teaching: What makes it Special? Journal of Teacher Education, 59, 389-407. doi: 10.1177/0022487108324456 
Carrillo, J. (1998). Modos de resolver problemas y concepciones sobre la matemática y su enseñanza: metodología de la investigación y relaciones. Huelva: Universidad de Huelva Publicaciones.

Carrillo, J., Flores, P., y Contreras, L.C. (2013). Un modelo de conocimiento especializado del profesor de matemáticas. En L. Rico, M.C. Cañadas, J. Gutiérrez, M. Molina \& I. Segovia (2013), Investigación en didáctica de la matemática. Homenaje a Encarnación Castro (pp. 193-200). Granada: Editorial Comares.

Climent, N., Romero-Cortés, J.M., Carrillo, J., Muñoz-Catalán, M.C., y Contreras, L.C. (2013). ¿Qué conocimientos y concepciones movilizan futuros maestros analizando un vídeo en el aula? Revista Latinoamericana de Investigación en Matemática Educativa, 16(1), 13-36.

Colera, J. y Gaztelu, I. (2007). Matemáticas 1ํㅡㄹ Eucación Secundaria, Andalucía. Madrid: Anaya.

Contreras, L. C. Concepciones de los profesores sobre resolución de problemas. Huelva: Universidad de Huelva Publicaciones.

Ernest, P. (1989). The impact of beliefs on the teaching of mathematics. En Keitel, C. et al. (Eds) Mathematics, Education and Society. Science and Technology Education. Document Series 35 (pp.99-101). Paris: Unesco.

Fernández-Gago, J y Carrillo, J. (2014). Cómo se esfuerzan los alumnos en resolución de problemas matemáticos (I). Bolema, 28(48), 149-169. doi: 10.1590.

Flores, P (1998). Concepciones y creencias de los futuros profesores sobre las matemáticas, su enseñanza y aprendizaje. Granada: Comares.

Gil, N., Blanco, L. y Guerrero, E. (2005). El dominio afectivo en el aprendizaje de las Matemáticas. Una revisión de sus descriptores básicos. Unión, Revista Iberoamericana de Educación Matemática 2, 15-32.

Jaworski, B \&Potari, D. (2002). Tackling complexity in mathematics teaching development: using the teaching triad as a tool for reflection and analysis. Journal of Mathematics Teacher Education, 4(5), 351-380. doi: 10.1023/A:1021214604230.

Leatham, K.R. (2006). Viewing mathematics teachers' beliefs as sensible systems. Journal of Mathematics Teacher Education, 9(1), 91-102.

Marina, J. A. (2004a). El misterio de la voluntad perdida. Barcelona: Anagrama.

Marina, J. A. (2004b). Teoría de la inteligencia creadora. Barcelona: Anagrama.

Marina, J. A. (2009) El laberinto sentimental. Barcelona: Anagrama.

Marina, J.A. (2012). La inteligencia ejecutiva. Barcelona: Ariel.

Marina, J.A. (2013) El aprendizaje de la creatividad. Barcelona. Ariel.

Monteiro, R., Carrillo, J. \& Aguaded, S. (2008). Emergent theorizations in Modelling the Teaching of Two Science Teachers. Research in Science Education, 38(3), 301-319. doi: 10.1007/s11165-007-9051-z. 
Merrian, S. (1988).Case study research in education. A qualitative approach. San Francisco: Jossey- Bass.

Muñoz-Catalán, M.C. (2012). El desarrollo profesional de una maestra novel. Un estudio de caso en un entorno colaborativo centrado en la enseñanza de las Matemáticas. Tomos (I-V). Saarbrücken (Alemania): Lambert Academic Publishing GmbH\&Co.KG.

Orden de 19 de agosto de 2007, por la que se desarrolla el currículo correspondiente a la Educación Secundaria Obligatoria en Andalucía.

O'shea \& Leavy(2013). Teaching mathematical problem-solving from an emergent constructivist perspective: the experience of Irish primary teachers. Journal Mathematical Teacher Education, 16, 293-318. doi: 10.1007/s10857-013-9235-6

Papini, M.C. (2003). Algunas explicaciones vigotskyanas para los primeros aprendizajes del álgebra. Revista Latinoamericana de Investigación en Matemática Educativa, 1(6), 41-71.

Polya, G. (1992). ¿Cómo plantear y resolver problemas? Princeton: University Pres. Traducción Editorial Trillas, México.

Puig, L. (1996) Elementos de resolución de problemas. Granada: Comares.

Raymond, A.M. (1997). Inconsistency between a beginning Elementary School teacher's Mathematic belief and teaching practice. Journal for Research in Mathematics Education, 28(5), 550-576.

Ribierio, C., Carrillo, J. \& Monteiro, R. (2009). Analyzing a teacher's practice from the relations between her cognitions. The contribution of a cognitive model to the understanding of what seems to be happing. Proceedings of 33rd Conference of International Group for the Psycologhy of Mathematics Education, vol 1, 455. Thessaloniki.

Saiz, I. y otros (2014). Sobre la complejidad de la gestion en una clase de matemática: entre lo planificado y la realidad del aula. Modelización algebraica de problemas planteados en Z. Educación Matemática, 26(1).

Schoenfeld, A. H. (1985). Mathematical problem solving. New York: Academic Press.

Schoenfeld, A. H. (1992). Learning to think mathematically: Problem solving, metacognition and sense making in mathematics. In: Grows, D. (Ed.). Handbook of Research on Mathematics Teaching and Learning, (pp. 334-370). New York: Macmillan.

Schoenfeld, A. H. (2000). Models of the teaching process. Journal of Mathematical Behaviour, 18(3), 243-261. doi: 10.1016/S0732-3123(99)00031-0

Schoenfeld, A. H., Minstrell, J. \& Van Zee, E. (2000). The detailed analysis of an established teacher's non-traditional lesson. Journal of Mathematical Behavior, 18(3), 281-325. doi:10.1016/S0732-3123(99)00035-8 
Schoenfeld, A.H: (2015). Whar counts, when? Reflections on beliefs, affect, attitude, orientations, habits of mind, grain size, time scale, context theory and method. In Birgit Pepin, Bettina Roesken-Winter, (Ed). From beliefs to dynamic affect systems in mathematics education (pp. 395-404). Trondheim, Norway: Springer. doi:10.1007/978-3-319-06808-4

Xenofontos, C. (2009). Towards a six-dimensional model n mathematics teachers' beliefs.. Proceedings of 33rd Conference of International Group for the Psycologhy of Mathematics Education, 5, 369-377. Thessaloniki.

Zimmerlin, D. \& Nelson, M (2000). The detailed analysis of a Beginning teacher carrying out of a traditional lesson. Journal of Mathematical Behaviour, 18(3) 263-279. doi: 10.1016/S0732-3123(99)00032-2

JOAQUÍN FERNÁNDEZ-GAGO

Dirección: Facultad de Ciencias de la Educación de Málaga, Bulevar Louis Pasteur 25, 29010 Málaga

Teléfono: +34952132617 


\section{ANEXO}

\section{jercicios y problemas}

\section{Ejercicio Iresuelto}

$$
\begin{aligned}
2(3 x+1)-x & =2-3(x-4) \\
6 x+2-x & =2-3 x+12 \\
x & =\frac{3}{2}
\end{aligned}
$$

$25 \square \square \square$ Halla $x$ en cada caso:

$$
\begin{aligned}
& \text { a) } 2(x+5)=16 \\
& \text { b) } 5=3 \cdot(1-2 x) \\
& \text { c) } 5(x-1)=3 x-4 \\
& \text { d) } 5 x-3=3-2(x-4) \\
& \text { e) } 10 x-(4 x-1)=5 \cdot(x-1)+7 \\
& \text { f) } 6(x-2)-x=5(x-1) \\
& \text { g) } 7(x-1)-4 x-4(x-2)=2 \\
& \text { h) } 3(3 x-2)-7 x=6(2 x-1)-10 x \\
& \text { i) } 4 x+2(x+3)=2(x+2)
\end{aligned}
$$

$26 \square \square$ Resuelve estas ecuaciones:

$$
\begin{array}{ll}
\text { a) } \frac{x-6}{2}=1 & \text { b) } \frac{x}{3}-1=2 \\
\text { c) } \frac{x}{5}+\frac{1}{5}=1 & \text { d) } \frac{x}{7}+\frac{2}{7}=x \\
\text { e) } 4=x+\frac{x}{3} & \text { f) } x=1-\frac{x}{5}
\end{array}
$$

\section{Problemas pard resolver con ecuaciones}

$27 \square \square \square$ Si triplicas un número y al resultado le restas 16, obtienes 29. ¿Cuál es el número?

28 mạ ¿Cuál es el número que sumado con su anterior y su siguiente da 117 ?
EL ANTERTOR $\longrightarrow x-1$
EL NÚMERO $\longrightarrow x$
EL POSTERIOR $\longrightarrow x+1$

$29 \square \square$ La suma de tres números consecutivos es 84 . ¿Qué números son?

30 wrt Si a un número le restas 28 unidades, obtienes el mismo resultado que si lo divides entre 3. ¿Qué número es?

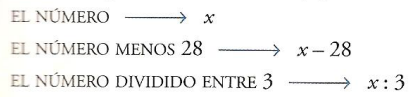

$31 \square \square \square$ Si a este cántaro le añadieras 13 litros de agua. tendría el triple que si le sacaras dos. ¿¿Cuántos litros de agua hay en el cántaro?

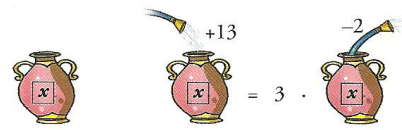

$32 \square \square \square$ En mi colegio, entre alumnos y alumnas somos 624. El número de chicas supera en 36 al de chicos. ¿Cuántos chicos hay? ¿Y chicas?

$$
\begin{gathered}
\text { CHICOS } \longrightarrow x \quad \text { CHICAS } \longrightarrow x+36 \\
\qquad \text { CHICOS }+ \text { CHICAS }=624
\end{gathered}
$$

$33 \square \square \square$ Sabiendo que un yogur de frutas es 5 céntimos más caro que uno natural, y que seis de frutas y cuatro naturales me han costado $4,80 €$, ¿cuánto cuesta un yogur natural? ¿Y uno de frutas?

NATURAL $\longrightarrow x € \quad$ FRUTAS $\longrightarrow(x+0,5) €$

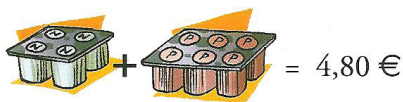

34 $\square \square \square$ Roberta tiene un año menos que su hermana Marta, y ya tenía cinco cuando nació Antonio, el más pequeño. ¿Cuál es la edad de cada uno, sabiendo que entre los tres, ahora, suman 35 ańos?

ROBERTA $\rightarrow x \quad$ MARTA $\rightarrow x+1 \quad$ ANTONIO $\rightarrow x-5$

$35 \square \square \square$ En una ferretería se venden clavos en cajas de tres tamaños diferentes. La caja grande contiene el doble de unidades que la mediana, y esta, el doble que la pequeña. Si compras una caja de cada tamaño, te llevas 500 unidades. ¿Cuántos clavos tiene cada caja?

$36 \square \square \square$ Un kilo de chirimoyas cuesta el doble que uno de naranjas.

Por tres kilos de chirimoyas y cuatro de naranjas se han pagado $11 €$. ¿¿A cómo están las unas y las otras?

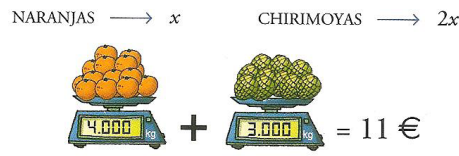

